\title{
Power Factor Corrector Based on Parallel Quasi- Resonant Pulse Converter with Fast Current Loop
}

\author{
Yuriy Denisov (Professor, Chernihiv State Technological University, Ukraine), \\ Serhii Stepenko (PhD Student, Chernihiv State Technological University, Ukraine)
}

\begin{abstract}
The problems, devoted to power quality and particularly power factor correction, are of great importance nowadays. The key requirements, which should be satisfied according to the energy efficiency paradigm, are not limited only by high quality of the output voltage (low total harmonic distortion), but also assume minimal power losses (high efficiency) in the power factor corrector (PFC). It could be satisfied by the use of quasi-resonant pulse converter (QRPC) due to its high efficiency at high switching frequency instead of the classical pulse-width modulated (PWM) boost converter. A dynamic model of QRPC with zero current switching (ZCS) is proposed. This model takes into account the main features of QRPC-ZCS as a link of a PFC closed-loop system (discreteness, sharp changes of parameters over switching period, input voltage impact on the gain). The synthesized model is also valid for conventional parallel pulse converter over an active interval of commutation. The regulator for current loop of PFC was synthesized based on digital filter using proposed model by the criterion of fast acting.
\end{abstract}

Keywords - Control system synthesis, closed loop systems, digital filters, power conversion, zero current switching.

\section{INTRODUCTION}

Due to the fast development of SmartGrid concept and since the number of renewable energy sources is continuously growing, the problems devoted to power quality are of great importance nowadays.

\section{A. General Overview}

For instance, some works are devoted to higher harmonic compensation in grid connected converter modes [1]. Higher harmonics increase power losses in transformers and transmission lines and can shorten their lifetime. Therefore, satisfying the power quality requirements (total harmonic distortion limits for current and voltage) is extremely urgent issue.

There are works which deal with power factor correction (PFC) circuits in the context of improvement of power supply for MicroGrids [2]. A number of works devoted to this topic show description, analysis and comparison of different power factor correctors for implementation in uninterruptable power supplies [3]. Special attention was paid to the efficiency of the correctors and possibility to build them utilizing unified power modules.

The current trend is to use the concept of interleaving for PFC circuits, for which the development recommendations are presented in [4]. Thus, a single converter is replaced by two or more parallel running converters which operate in phase.
Therefore the current ripples at the output are mutually compensated which reduces the requirements for filtration. Other advantages of this approach are to provide modularity, heat dissipation, as well as opportunities to optimize a price/performance ratio. Of course, there are certain drawbacks such as greater number of components and a more complex control system.

In [5] different topologies are presented for providing continuous conduction mode (continuous input current) and discontinuous conduction mode (discontinuous input current). The paper [6] provides an overview of boost converters with a fixed frequency PWM operating in continuous conduction mode.

\section{B. Problem Discussion}

Actually, the main parameters of the PFC performance are the efficiency and power factor, which depend on the switching frequency. In [7], an integrated approach to the implementation of the PFC is considered, that takes into account the power factor, harmonic distortion and the efficiency of the converter. The efficiency of the use of feedback from the output voltage is shown. Some works are devoted to the estimation of semiconductor switching losses under hard switching [8]. But the modern approach is to use soft switching for the reduction of the power losses.

The paper [9] provides an overview of methods for active power factor correction, used for single-phase circuits. It is focused on the implementation of zero voltage switching and zero current switching, as well as the use of snubbers. In [10], the basic schemes of PFC, as well as control features for use in three-phase inverters are discussed. The possibilities of providing soft switching are presented, among which are resonant, quasi-resonant, multiresonant converters, circuits with quasi-rectangular pulse-width modulation (PWM) and others.

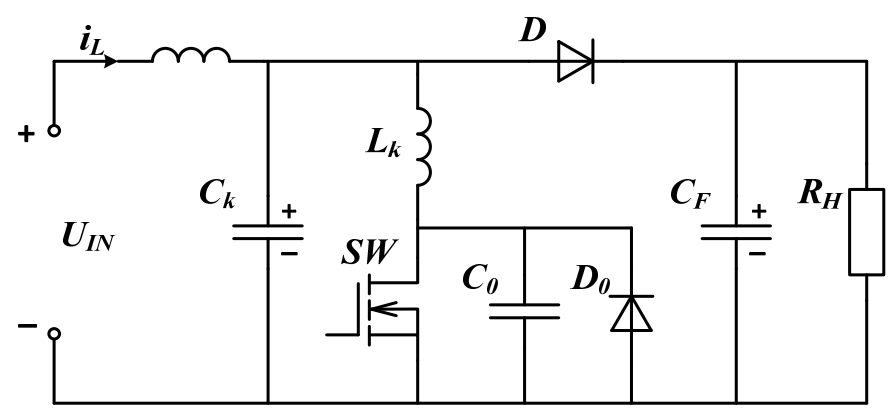

Fig. 1. Parallel quasi-resonant pulse converter with zero current switching. 
Implementation of quasi-resonant pulse converters (QRPC) with zero current switching (ZCS) into PFCs allows to reduce power losses maintaining high efficiency at high switching frequency and obviously improve power factor (Fig. 1).

However, high switching frequency raises the problem of improving the speed of the current loop in PFC. The main task of the current loop is to ensure a minimal deviation of the input current regarding to the reference sine wave in real time. Therefore, the problem of high-speed PFC current loop synthesis appears.

The known PFC circuits are usually performed on the basis of conventional parallel pulse converters. In their current loops non-optimal regulators are used based on so-called reversecurrent amplifiers. To improve efficiency of PFC at high frequency in [11] multiphase pulse converters are used. They are critical in terms of extra expenses as well as from the point of view of ensuring an adequate stability reserve of the closedloop system.

The processes in single-loop PFC with conventional parallel pulse converter with zero-current turning on switch are investigated using a method of averaging in [12]. In other words, transistor works in boundary conduction mode (BCM), i.e. it is on the border between continuous conduction mode (CCM) and discontinuous conduction mode (DCM). In our view, such decision yields the way of using QRPC-ZCS, in which high-frequency current ripple and turn off power losses are minimal. In this case, the negative impact of the QRPCZCS variable switching frequency on the filtering quality of input current harmonics can be reduced to minimum due to its increase up to several $\mathrm{MHz}$.

\section{Unsolved Aspects of the Problem}

The feature of structural building of the known PFCs is that in them a voltage loop is the main. In these structures the product of the voltage loop error and the reference sine current comes to the input of the subordinate current loop, thus causing a distortion. It should be noted that the reference current in PFC follows a rectified supply voltage. Due to the number of various switching processes affecting the shape of supply voltage the reference current usually is not accurate.

The precision of the closed-loop system can not exceed the accuracy of the reference signal. Therefore, the improvement of PFC dynamic accuracy, which has a positive impact on its energy performance, can be achieved by improving the accuracy of the reference current signal as well as by the rational structural construction of the control system.

In addition, the general problem of improvement of PFC energy efficiency was not sufficiently addressing the issues of PFC construction on the basis of parallel QRPC-ZCS working in $\mathrm{MHz}$ range. Also the issues of speed improvement of current loop were virtually neglected. The maximum effect of the application of parallel high-frequency pulse converters and QRPC in PFC is very difficult to achieve without the solutions to the mentioned issues.

\section{Purpose of This Work}

The aim of this work is to develop a dynamic model of parallel QRPC-ZCS and design the optimal regulator by the criterion of fast acting for the current loop of high-frequency converter, which is the main loop in the structure of PFC.

\section{DYNAMIC MODEL DESCRIPTION}

Consider the parallel QRPC-ZCS as a part of a closed-loop system of PFC. It has such particular qualities: discreteness, frequency modulation (FM), which may be first-order or second-order depending on specifics of the control system, a rapid change of the parameters on the switching interval. Obviously, QRPC-ZCS as a part of a closed-loop system has a complex discrete non-linearity, which creates certain problems in its analysis and optimization.

In addition to the mentioned features, which are conventional for FM blocks, there is one specific characteristic for QRPC-ZCS. It is related to its internal structural imbalance that appears when duty cycle $\gamma \geq 0.5$.

Besides, there is another specific feature for QRPC-ZCS working in the structure of PFC. It is the fact that its input signal is rectified (unfiltered) voltage with frequency $100 \mathrm{~Hz}$. This feature leads to the dependence of QRPC-ZCS gain as a part of the system from input voltage changes. In addition to the high-frequency ripple and fundamental frequency of the rectified voltage, subharmonic frequencies may occur in the input voltage spectrum [13]. One of the reasons for their occurrence is the discreteness of the converter.

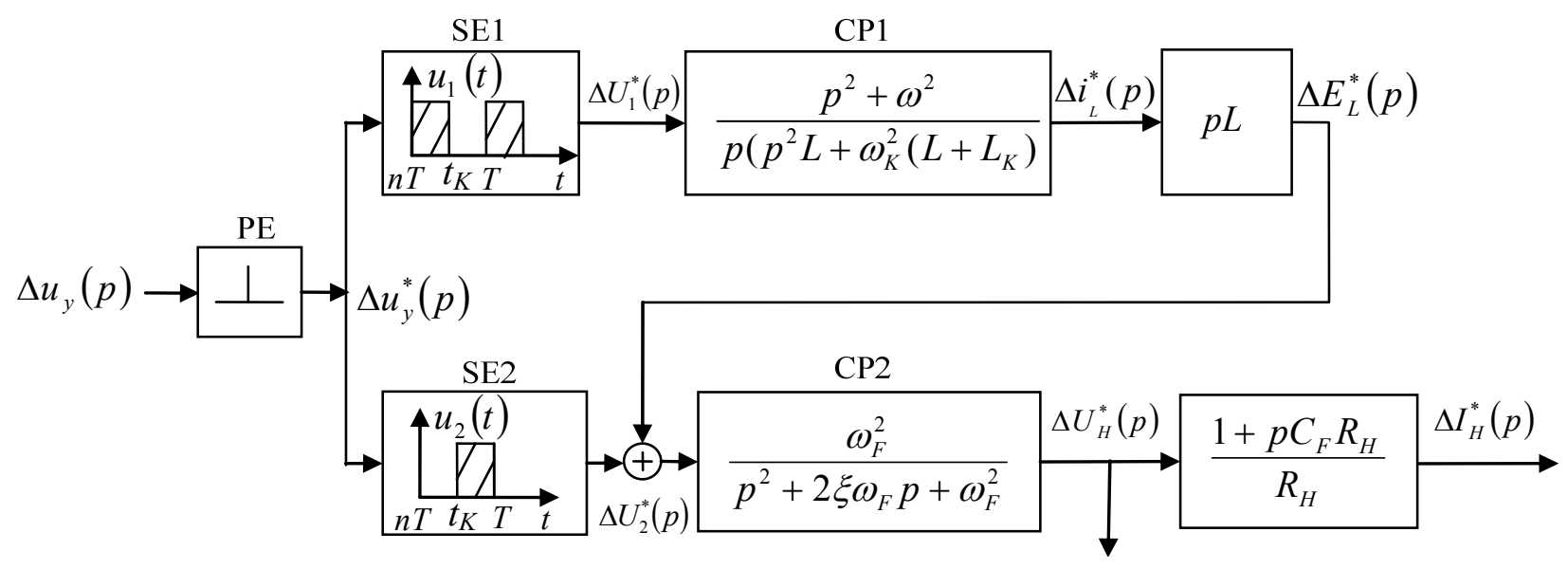

Fig. 2. Proposed dynamic model of quasi-resonant pulse converter with zero-current switching. 
Therefore, some assumptions and idealization of certain factors within the development of a dynamic model of QRPCZCS are inevitable in such cases.

Particularly, assuming that there is implemented a first order FM in the system, with duty cycle $\gamma \leq 0.5$; the elements of QRPC-ZCS are ideal; power supply is an ideal voltage source; the PFC switching frequency is changing slowly. We also assume that the process of regulation is going under infinitely small deviations of the state variables from their values in a static mode. The influence of parasitic parameters of the circuit elements is neglected.

Upon the results of analysis of electromagnetic processes in parallel QRPC-ZCS [14] and the assumptions made, its dynamic model is proposed (Fig. 2). Discreteness of the system is taken into account by the pulse element PE, which works under infinitely small deviations of the switching frequency from the static mode. Shaping elements SE1, SE2 consider the sharp change of parameters on the separate intervals of the switching period $T=1 / f_{K}$, where $f_{K}$ is switching frequency.

For some random switching period over capacitor recharge time interval $n T \leq t \leq n T+t_{i}$ converter input voltage is $u_{1}(t)$, which is a "cut-out" of the rectified voltage ( $t_{i}$ is the i-th pulse duration).

$u_{1}(t)$ image in the frequency domain over interval $t$ can be found using convolution theorem as product of a sine function image $f_{1}(p)=E \omega /\left(p^{2}+\omega^{2}\right)$ and a rectangular single pulse image $f_{2}(p)=\left(\exp (-p n T)+\exp \left(-p\left(n T+t_{i}\right)\right)\right) / p$. In such a way $u_{1}(p)$ was derived as shown in (1).

$$
\begin{array}{r}
u_{1}(p)=E\left[\left(e^{-p t_{1}} \sin \omega t_{1}-e^{-p t_{2}} \sin \omega t_{2}\right) p+\left(e^{-p t_{1}} \cos \omega t_{1}-\right.\right. \\
\left.\left.-e^{-p t_{2}} \cos \omega t_{2}\right) \omega\right] /\left(p^{2}+\omega^{2}\right),
\end{array}
$$

where the borders of the interval are $t_{1}=n T$ and $t_{2}=n T+t_{i}$, resonant circuit recharge time is $t_{i}=2 \pi / \omega_{K}$ and resonant angular pulsation is $\omega_{K}=1 / \sqrt{L_{K} C_{K}}$, that corresponds to the switching frequency $f_{K}, \omega$ is the grid frequency, $L$ is input inductance, $L_{K}$ is resonant circuit inductance, $C_{K}$ is resonant circuit capacitance.

The image representation (1) of the voltage applied to the input of QRPC-ZCS allows us to take into account the effect of an additional second order pulse-amplitude modulation (PAM II), which is accompanied by FM.
Thus, strictly speaking, there is a combined modulation in QRPC-ZCS. On the switching interval, which is characterized by the presence of SE1, continuous part CP1 is passive, as recharge processes of the switching capacitor does not directly affect the processes in load. Obviously, in QRPC-ZCS (even with second order FM) control process a delay occurs. It is determined by the resonant circuit recharge time.

Considering the effect of PAM II, using (1), the system analysis becomes more complicated and would be difficult to achieve.

At high switching frequency, as considered in this paper, it can be assumed without appreciable error, that the passive switching interval (the duration of which is a fraction of microseconds) satisfies the following equation (2):

$$
u_{1}(n T) \cong u_{1}\left(n T+t_{i}\right) .
$$

Then (3) could be derived:

$$
U_{1}(p)=U(n)\left(e^{-p t_{1}}-e^{-p t_{2}}\right) / p ，
$$

where $U(n)=E \sin \bar{\omega} n, \bar{\omega}=\omega T, n=0,1,2, \ldots N, N$ is the number of switching periods over one period of the rectified voltage at the input of QRPC-ZCS.

Since capacitor $C_{K}$ is recharged, some conditions for the active switching time interval $n T+t_{i} \leq t \leq(n+1) T$ appear [14]. In this case, in the input inductor arises electromotive force (EMF) of self-induction. Its image in the frequency domain is $E_{L}(p)=i_{1}(p) p L$, where

$$
i_{1}(p)=\frac{u_{1}(p)\left(p^{2}+\omega^{2}\right)}{p\left(p^{2} L+\omega_{K}^{2}\left(L+L_{K}\right)\right)} .
$$

Considering $L>>L_{K},(5)$ could be derived:

$$
E_{L}(p) \cong u_{1}(p) \text {. }
$$

This EMF is added to the voltage existing at the output of SE2. The addition is performed by an adder (Fig. 2), the output voltage of which is supplied to the load. The image of this voltage (at the second switching interval) is shown in (6):

$$
U_{2}^{*}(p)=\left[U_{1}(p)+E_{L}(p)\right] * W_{C P 2}(p) .
$$

The transfer function of the continuous part of voltage loop CP2 is represented by (7).

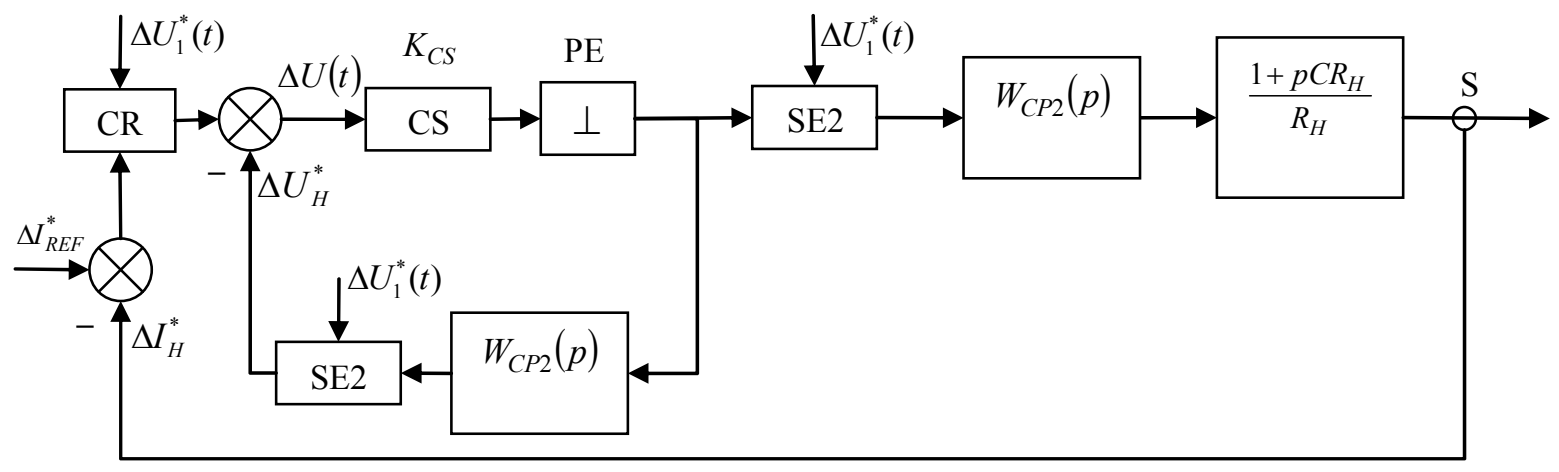

Fig. 3. Closed-loop automatic control system of power factor corrector for active interval. 


$$
W_{C P 2}(p)=\frac{\omega_{F}^{2}}{p^{2}+2 \xi \omega_{F} p+\omega_{F}^{2}}
$$

where

$$
\omega_{F}=\frac{1}{\sqrt{L C_{F}}}, \xi=\frac{\rho}{2 R_{H}}, \rho=\sqrt{\frac{L}{C_{F}}},
$$

$R_{H}$ is load resistance, $C_{F}$ is filter capacitance.

\section{Closed-Loop Automatic Control System of PFC}

The block diagram of a closed-loop automatic control system (ACS) of PFC for active interval is shown in Fig. 3. It supports the principle of a slave control with current loop as the main control loop of the system.

In the shown block diagram (Fig. 3) CR is current regulator, $\mathrm{CS}$ is control system, $U_{1}(t)$ is the output voltage of PFC rectifier, $\mathrm{S}$ is the current sensor.

The block diagram of ACS in Fig. 3 corresponds to the infinitely small deviations of the output parameters from their values in the static mode, i.e. discrete values $\Delta U_{H}^{*}(t), \Delta U^{*}(t), \Delta U_{1}^{*}(t), \Delta I_{R E F}^{*}(t), \Delta I_{H}^{*}(t)$ correspond to infinitely small values of the disturbances. Such system is valid for conventional parallel pulse converter as well. The made assumptions allow the use of linear pulse systems theory for the process optimization in the PFC current loop by the criterion of fast acting with setting up the processes for a finite duration [15].

To accomplish that task, we will find the discrete transfer function of an open-loop system (Fig. 3) for the moment of pulse element switching. First of all, we will define the openloop transfer functions of voltage loop and current loop.

Considering the voltage loop at the n-th active interval $\left(t_{1}=n T+t_{K} ; t_{2}=(n+1) T\right.$, where $t_{K}$ is the $\mathrm{k}$-th pulse duration as shown in Fig. 2) one can write:

$$
W_{p H}^{*}(z)=Z_{M}\left[2 U(n) \frac{e^{-p t_{1}}-e^{-p t_{2}}}{p} * \frac{\omega_{F}^{2}}{p^{2}+2 \xi \omega_{F} p+\omega_{F}^{2}}\right] .
$$

After modified Z-transform (9) is performed, we obtain the transfer function of an open voltage loop (10).

$$
W_{p H}^{*}(z, \varepsilon)=2 U(n) \frac{B_{1}^{H} z^{2}-B_{2}^{H} z+B_{3}^{H}}{z^{2}-2 z e^{-a} \cos \bar{\omega}_{0}+e^{-2 a}}, \gamma \leq \varepsilon \leq 1,
$$

where

$$
\begin{gathered}
B_{1}^{H}=1+2 A_{H} e^{-a(\varepsilon-\gamma)} \cdot \cos \left[\bar{\omega}_{0}(\varepsilon-\gamma)-\psi_{H}\right], \\
B_{2}^{H}=2 e^{-a} \cos \bar{\omega}_{0}+2 A_{H}\left[e^{-a \varepsilon} \cdot \cos \left(\bar{\omega}_{0} \varepsilon-\psi_{H}\right]+\right. \\
\quad+e^{-a(1-\gamma+\varepsilon)} \cdot \cos \left[\bar{\omega}_{0}(\varepsilon-\gamma-1)-\psi_{H}\right], \\
B_{3}^{H}=e^{-2 a}+2 A_{H} \cdot e^{-a(1+\varepsilon)} \cdot \cos \left[\bar{\omega}_{0}(\varepsilon-1)-\psi_{H}\right], \\
\bar{\omega}_{0}=\bar{\omega}_{F} \sqrt{1-\xi^{2}}, \bar{\omega}_{F}=\omega_{F} T, a=\xi \bar{\omega}_{F}, \gamma=\frac{t_{K}}{T} \\
\psi_{H}=\operatorname{arctg} \frac{a}{\bar{\omega}_{0}}, A_{H}=\frac{\bar{\omega}_{F}}{2 \sqrt{1-\xi^{2}} \cdot \sqrt{\bar{\omega}_{F}^{2}+a^{2}}} .
\end{gathered}
$$

The transfer function of the closed voltage loop for the moment of pulse element switching is shown in (16):

$$
W_{3 H}^{*}(z, 1)=\frac{K_{C S}}{1+K_{C S} \cdot z^{-1} W_{p H}^{*}(z, 1)} .
$$

As a result of transformations we obtain (17):

$W_{3 H}^{*}(z, 1)=K_{C S} \frac{z\left(z^{2}-2 z e^{-a} \cos \bar{\omega}_{0}+e^{-2 a}\right)}{z\left(z^{2}-2 z e^{-a} \cos \bar{\omega}_{0}+e^{-2 a}\right)+K_{0}\left(B_{11}^{H} z^{2}-B_{21}^{H} z+B_{31}^{H}\right)}$,

where $K_{0}=2 U(n) K_{C S}$ and coefficients $B_{11}^{H}, B_{21}^{H}, B_{31}^{H}$ were obtained from corresponding equations (11), (12), (13) by substitution $\varepsilon=1$.

The transfer function of the open current loop can be found by performing modified Z-transform with the transfer function of its continuous part (18):

$W_{p T}(z, \varepsilon)=Z_{M}\left[K_{S} \frac{e^{-p t_{1}}-e^{-p T}}{p} \cdot \frac{\omega_{F}^{2}\left(1+p R_{H} C_{F}\right) \cdot 2 U(n)}{R_{H}\left(p^{2}+2 \xi \omega_{F} p+\omega_{F}^{2}\right)}\right]$,

where $K_{S}$ is current sensor gain.

Considering the moment of pulse element switching we obtain (19):

$$
W_{p T}^{*}(z, 1)=2 U(n) \frac{\left(B_{11}^{T} z^{2}-B_{21}^{T} z+B_{31}^{T}\right) K_{S}}{R_{H} z\left(z^{2}-2 z e^{-a} \cos \bar{\omega}_{0}+e^{-2 a}\right)} .
$$

In (19) coefficients $B_{11}^{T}, B_{21}^{T}, B_{31}^{T}$ were obtained from corresponding equations (11), (12), (13) by substitution $\varepsilon=1$ and replacing $A_{H}, \psi_{H}$ from (15) by corresponding values (20) and (21):

$$
\begin{gathered}
A_{T}=\frac{\bar{\omega}_{F}^{2} \sqrt{\bar{\omega}_{0}^{2}+\left(\bar{\omega}_{0}^{2} \bar{T}_{1}-a+a^{2} \bar{T}_{1}\right)^{2}}}{2 \bar{\omega}_{0}\left(a^{2}+\bar{\omega}_{0}^{2}\right)}, \\
\psi_{T}=\operatorname{arctg} \frac{\bar{\omega}_{0}^{2} \bar{T}_{1}-a+a^{2} \bar{T}_{1}}{\bar{\omega}_{0}},
\end{gathered}
$$

where $\bar{T}_{1}=\frac{C_{F} R_{H}}{T}$.

The transfer function of the open-loop PFC is following:

$$
W_{p}^{*}(z, 1)=W_{3 H}^{*}(z, 1) \cdot W_{p T}^{*}(z, 1) \text {. }
$$

Taking into account (17) and (19) one can write:

$W_{p}^{*}(z, 1)=$

$$
=\frac{K_{0} \cdot\left(B_{11}^{T} z^{2}-B_{21}^{T} z+B_{31}^{T}\right) \cdot K_{S}}{R_{H}\left[z^{3}-z^{2}\left(2 e^{-a} \cos \bar{\omega}_{0}-K_{0} B_{11}^{H}\right)+z\left(e^{-2 a}-B_{21}^{H} K_{0}\right)+K_{0} B_{31}^{H}\right]} \cdot
$$

In the transfer function of the open-loop PFC $K_{0}$ depends on the voltage $U(n)$ that is applied to the input of QRPC-ZCS. It causes the dependence of the steady-state control deviation from this voltage. We will define the influence of the input voltage on the steady-state control deviation.

The transfer function of PFC by control deviation:

$$
W_{\varepsilon}^{*}(z, 1)=\frac{1}{1+W_{p}^{*}(z, 1)} .
$$

Taking into account (23) one can write:

$$
W_{\varepsilon}^{*}(z, 1)=\frac{z^{3}+z^{2} a_{2}^{H}+z a_{1}^{H}+a_{0}^{H}}{z^{3}+z^{2} a_{2}^{H}+z a_{1}^{H}+a_{0}^{H}+K_{0}^{T}\left(B_{11}^{T} z^{2}-B_{21}^{T} z+B_{31}^{T}\right)},
$$


where

$$
\begin{aligned}
a_{0}^{H} & =K_{0} B_{31}^{H}, \\
a_{1}^{H} & =e^{-2 a}-B_{21}^{H} K_{0}, \\
a_{2}^{H} & =-2 e^{-a} \cos \bar{\omega}_{0}+K_{0} B_{11}^{H}, \\
K_{0}^{T} & =\frac{K_{0} \cdot K_{S}}{R_{H}} .
\end{aligned}
$$

A steady-state control deviation of PFC system exposed by input single step:

$$
\varepsilon(n)=\lim _{z \rightarrow 1}\left\{\frac{z-1}{z} \cdot \frac{1}{1+W_{p}(z, 1)} \cdot \frac{z}{z-1}\right\} .
$$

After corresponding conversions we obtain:

$$
\varepsilon(n)_{S T}=\frac{1+a_{2}^{H}+a_{1}^{H}+a_{0}^{H}}{1+a_{2}^{H}+a_{1}^{H}+a_{0}^{H}+K_{0}^{T}\left(B_{11}^{T}-B_{21}^{T}+B_{31}^{T}\right)} .
$$

Taking into account (29) it is obvious, that input voltage increasing leads to reducing the steady-state control deviation:

$$
K_{0}^{T}=\frac{2 U(n) K_{S} K_{C S}}{R_{H}} .
$$

By the method of finite duration processes adjustment [15] we will synthesize the regulator of PFC current loop by the criterion of fast acting. From (23) one can write:

$$
\begin{array}{r}
P^{*}(z, 1)=K_{0}^{T}\left(B_{11}^{T} z^{2}-B_{21}^{T} z+B_{31}^{T}\right), \\
Q^{*}(z, 1)=\left(z^{3}-z^{2}\left(2 e^{-a} \cos \bar{\omega}_{0}-K_{0} B_{11}^{H}\right)+z\left(e^{-2 a}-\right.\right. \\
\left.\left.-B_{21}^{H} K_{0}\right)+K_{0} B_{31}^{H}\right) .
\end{array}
$$

The minimum number of switching periods for which the process in PFC current loop can be established:

$$
S_{\min }=l_{Q}+r-1 \text {, }
$$

where $l_{Q}$ is the order of the polynomial $Q^{*}(z, 1), \mathrm{r}=1$ is specified astatizm order. As a result, we obtain that it is possible to establish a process in current loop for three switching periods $S_{\min }=3$. This goal is achieved when the following condition (33) is satisfied:

$$
P^{*}(z, 1) \cdot M^{*}(z, 1)+(z-1)^{r} \cdot N^{*}(z, 1)=z^{3},
$$

where $M^{*}(z, 1), N^{*}(z, 1)$ are some polynomials with corresponding orders $l_{M}, l_{N}, l_{M}=r-1=0, l_{N} \geq l_{p}, l_{p}$ is the order of polynomial $P^{*}(z, 1)$. In our case for $l_{p}=2$ we obtain:

$$
M^{*}(z, 1)=b_{0} ; \quad N^{*}(z, 1)=C_{2} z^{2}+C_{1} z+C_{0} .
$$

Assuming (34) we can modify (33) and one can write:

$$
K_{0}^{T}\left(B_{11}^{T} z^{2}-B_{21}^{T} z+B_{31}^{T}\right) b_{0}+\left(C_{2} z^{2}+C_{1} z+C_{0}\right)(z-1)=z^{3} .
$$

By equating the coefficients of the same powers of $z$ we can obtain the following equations:

$$
\begin{aligned}
& b_{0} K_{0}^{T} B_{11}^{T}+C_{1}-C_{2}=0, \\
& -b_{0} K_{0}^{T} B_{21}^{T}+C_{0}-C_{1}=0, \\
& b_{0} K_{0}^{T} B_{31}^{T}-C_{0}=0, \\
& C_{2}=1 .
\end{aligned}
$$

After their solution we can find:

$$
\begin{aligned}
& b_{0}=\frac{1}{K_{0}^{T} \cdot F\left(B^{T}\right)}, C_{0}=\frac{B_{31}^{T}}{F\left(B^{T}\right)}, C_{1}=\frac{B_{31}^{T}-B_{21}^{T}}{F\left(B^{T}\right)}, \\
& C_{2}=1, F\left(B^{T}\right)=B_{11}^{T}-B_{21}^{T}+B_{31}^{T} .
\end{aligned}
$$

The optimal transfer function of the correcting circuit in PFC current loop in case of its serial connection is as the following:

$$
K_{K T}^{*}(z, 1)=\frac{Q^{*}(z, 1) \cdot M^{*}(z, 1)}{(z-1) \cdot\left(C_{2} z^{2}+C z_{1}+C_{0}\right)} \cdot
$$

By substitution values of $Q^{*}(z, 1), M^{*}(z, 1)$ into (38) we obtain:

$$
K_{K T}^{*}(z, 1)=\frac{b_{0}\left(z^{3}+a_{2}^{H} z^{2}+a_{1}^{H} z+a_{0}^{H}\right)}{(z-1) \cdot\left(C_{2} z^{2}+C_{1} z+C_{0}\right)} .
$$

The optimal transfer function of the closed-loop PFC is the following:

$$
W_{3 \text { opt }}^{*}(z, 1)=P^{*}(z, 1) \frac{M^{*}(z, 1)}{z^{3}} .
$$

Taking into account the values of $P^{*}(z, 1), M^{*}(z, 1)$ we obtain (41), which does not depend on input voltage.

$$
W_{3 \text { opt }}^{*}(z, 1)=\frac{B_{11}^{T} z^{2}-B_{21}^{T} z+B_{31}^{T}}{z^{3} \cdot F\left(B^{T}\right)} .
$$

The image of the output current under exposure of single step is the following:

$$
\begin{aligned}
I^{*}(z, 1)=W_{3 \text { opt }}^{*}(z, 1) \cdot \frac{z}{z-1}=\frac{B_{11}^{T}}{F\left(B^{T}\right) \cdot(z-1)} & -\frac{B_{21}^{T}}{F\left(B^{T}\right) \cdot z \cdot(z-1)}+ \\
& +\frac{B_{31}^{T}}{F\left(B^{T}\right) \cdot z^{2} \cdot(z-1)} .
\end{aligned}
$$

After conversion the image to the original we get:

$$
I^{*}(n, 1)=\frac{B_{11}^{T}}{F\left(B^{T}\right)}[n-1]-\frac{B_{21}^{T}}{F\left(B^{T}\right)}[n-2]+\frac{B_{31}^{T}}{F\left(B^{T}\right)}[n-3],
$$

or

$$
I^{*}(n, 1)=\left[\begin{array}{c}
\frac{B_{11}^{T}}{F\left(B^{T}\right)}, n=1 ; \\
\frac{B_{11}^{T}-B_{21}^{T}}{F\left(B^{T}\right)}, n=2 ; \\
\frac{B_{11}^{T}-B_{21}^{T}+B_{31}^{T}}{F\left(B^{T}\right)}=1, n=3 .
\end{array}\right.
$$

Obviously, the process in the PFC system ends at three intervals. It should be noted, that for certain values of the parameters the process ending in a finite number of intervals can have a large overshoot.

To overcome that, duration of the transition process should be increased. In this case, the degrees of polynomials $M^{*}(z, 1), N^{*}(z, 1)$ are increasing up to the desired value. 


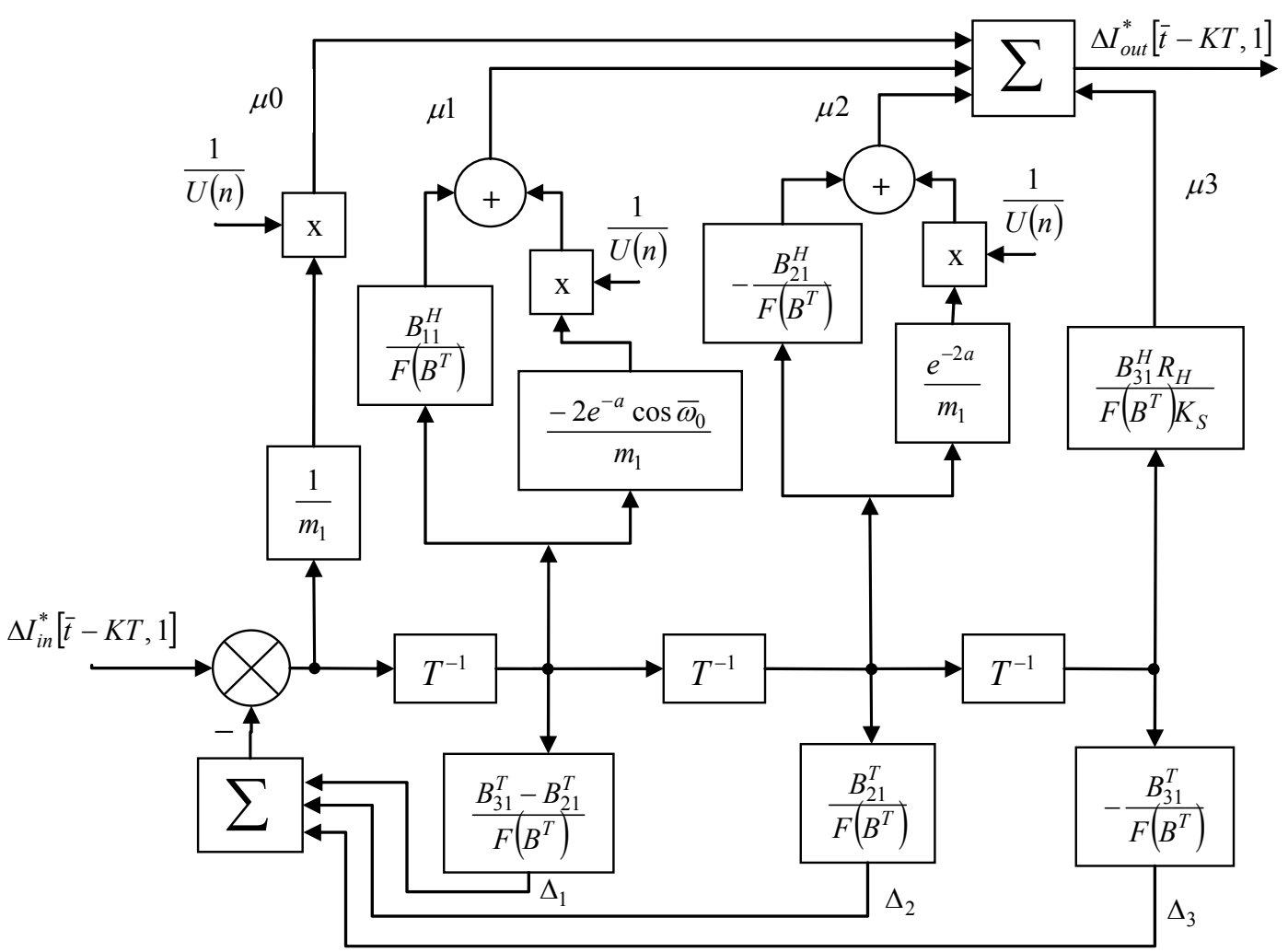

Fig. 4. Block diagram of the proposed discrete-continuous recursive digital filter.

Dividing the numerator and denominator of (39) by $z^{3}$ we obtain:

$K_{K T}^{*}(z, 1)=\frac{\Delta I_{\text {out }}^{*}(z, 1)}{\Delta U_{\text {in }}^{*}(z, 1)}=\frac{b_{0}\left(1+a_{2}^{H} z^{-1}+a_{1}^{H} z^{-2}+a_{0}^{H} z^{-3}\right)}{C_{2}+z^{-1}\left(C_{1}-C_{2}\right)+z^{-2}\left(C_{0}-C_{1}\right)-z^{-3} C_{0}}$

The resulting transfer function of the correcting circuit in the PFC current loop comply the following difference equation:

$\Delta I_{\text {out }}^{*}[t-K T, 1]=\sum_{K=0}^{3} \Delta I_{\text {in }}^{*}[t-K T, 1] \cdot \mu_{K}-\sum_{K=1}^{3} \Delta I_{\text {out }}^{*}[t-K T, 1] \cdot \Delta_{K}$,

$\mu_{0}=b_{0}, \mu_{1}=b_{0} a_{2}^{H}, \mu_{2}=b_{0} a_{0}^{H}, \Delta_{1}=C_{1}-C_{2}, \Delta_{2}=C_{0}-C_{1}, \Delta_{3}=-C_{0}$.

Taking into account (37) one can write:

$$
\begin{aligned}
& \mu_{0}=\frac{1}{U(n) m_{1}} ; \mu_{1}=\frac{B_{11}^{H}}{F\left(B^{T}\right)}-\frac{2 e^{-a} \cdot \cos \bar{\omega}_{0}}{U(n) m_{1}} ; \\
& \mu_{2}=-\frac{B_{21}^{H}}{F\left(B^{T}\right)}+\frac{e^{-2 a}}{U(n) m_{1}} ; \mu_{3}=\frac{B_{31}^{H} \cdot R_{H}}{F\left(B^{T}\right) K_{S}} ; \\
& \Delta_{1}=\frac{B_{31}^{T}-B_{21}^{T}}{F\left(B^{T}\right)} ; \Delta_{2}=\frac{B_{21}^{T}}{F\left(B^{T}\right)} ; \Delta_{3}=-\frac{B_{31}^{T}}{F\left(B^{T}\right)} ; \\
& m_{1}=\frac{2 K_{C S} K_{S} F\left(B^{T}\right)}{R_{H}} .
\end{aligned}
$$

The difference equation (46) corresponds to a discretecontinuous recursive filter, the block diagram of which is shown in Fig. 4. It is composed of adders, delay elements, as well as weight units in forward and backward links.
The correcting circuit (39) may be implemented in an analog form. To perform this, the W-transformation is needed with the transition from real frequency to pseudo frequency [13].

In case of digital implementation this dependence is taken into account by the presence of the ADC which converts the input voltage into a digital code. The resulting code is read with a frequency equal to the switching frequency of the PFC power switch. Then the code goes to one input of multiplier, the second input of which receives the code of the corresponding forward link coefficient that is shown in Fig. 4.

As an example, we calculate the parameters of the forward and reverse links of the optimal regulator in PFC current circuit for the following conditions: switching frequency $f=10^{5} \mathrm{~Hz}$, filter capacitance $C_{F}=10^{-4} \mathrm{~F}$, inductance of the input choke $L=10^{-3} H$, duty cycle $\gamma=0,4$, gain of control system $K_{C S}=1$ and current sensor $K_{S}=1$, load resistance $R_{H}=10$ Ohms, amplitude the rectified voltage $U_{M}=\sqrt{2} \cdot 220 \mathrm{~V}$.

Using (47) we can obtain:

$$
\begin{array}{r}
\mu_{0}=\frac{2,1}{U(n)} ; \mu_{1}=-\frac{8,3}{U(n)}+0,827 ; \mu_{2}=\frac{4,19}{U(n)}-1,68 ; \mu_{3}=8,27 ; \\
\Delta_{1}=-13,2 ; \Delta_{2}=26,8 ; \Delta_{3}=-13,6 .
\end{array}
$$

For $U(n)=1 \mathrm{~V}$, the steady-state control deviation of PFC system $\varepsilon_{S T}=2$. For $U(n)=U_{M}$, the steady-state control deviation of PFC system $\varepsilon_{S T}=6,3 \cdot 10^{-3}$.

These results show that $\mu_{0}$, as well as the first summands of $\mu_{1}, \mu_{2}$ for small values of the input voltage will have a significant impact on the process of correction, however, their impact will be negligible when $U(n)$ increase. 
We can use the average values of these coefficients, taking into account only the average value of the voltage applied to the input of PFC $U_{0}=2 E_{M} / \pi=202 \mathrm{~V}$.

In such case:

$$
\mu_{0 a v}=1,05 \cdot 10^{-2} ; \mu_{1 a v}=-4,15 \cdot 10^{-2}+0,827 ; \mu_{2 a v}=2,09 \cdot 10^{-2}-1,68 \text {. }
$$

When taking into account only the average values of the direct link coefficients, the correcting unit in PFC current loop is conveniently designed using analog components while neglecting the accuracy of regulation.

\section{CONCLUSIONS}

In this work a dynamic model of quasi-resonant pulse converter (QRPC) with zero-current switching (ZCS) is proposed, which is valid for conventional parallel pulse converter as well.

This model takes into account the main features of QRPCZCS as a link of a PFC closed-loop system (discreteness, sharp changes of parameters over switching period, input voltage impact on the gain).

Consideration of discreteness allowed synthesizing a digital current loop regulator by the criterion of fast acting. It takes into account the impact of QRPC-ZCS parameters, its input voltage and load on the parameters of the forward and backward links.

The further work is planned to be devoted to the validation of the proposed dynamic model of QRPC-ZCS by simulation applied to a given case, which will complete the provided mathematical analysis. The stability of the system after applying the proposed regulator with digital filter will be discussed and checked for different conditions by simulation in Matlab/Simulink as well.

\section{REFERENCES}

[1] Wrona, G. \& Jasinski, M. (2013). AC-DC Converter with Asymmetrical Higher Harmonics Compensation Function in Sustainable AC Grid. Electrical, Control and Communication Engineering, 2(1), pp. 5-13. Retrieved 6 Aug. 2013, from doi:10.2478/ecce-2013-0001

[2] A. Suzdalenko, A. Stepanov and I. Galkin, "Choice of power factor corrector for effective operation of MicroGrid and its elements," in Proceedings of International School on Nonsinusoidal Currents and Compensation (ISNCC), June 15-18, 2010, Lagow, Poland, pp. 234-238.

[3] I. Galkin, A. Stepanov, P. Suskis, "Selection of power factor corrector for modular uninterruptable power supply system," in Proceedings of 14th International Power Electronics and Motion Control Conference (EPE-PEMC), 6-8 Sept., 2010, Ohrid, Macedonia, pp.T13-17-T13-21.

[4] T. Nussbaumer, K. Raggl, and J.W. Kolar, "Design Guidelines for Interleaved Single-Phase Boost PFC Circuits," IEEE Transactions on Industrial Electronics, vol.56, no.7, pp.2559-2573, July 2009.

[5] Chongming Qiao and K.M. Smedley, "A topology survey of singlestage power factor corrector with a boost type input-current-shaper," IEEE Transactions on Power Electronics, vol.16, no.3, pp.360-368, May 2001.

[6] Z. Lai, K.M. Smedley, "A family of continuous-conduction-mode power-factor-correction controllers based on the general pulse-width modulator," IEEE Transactions on Power Electronics, vol.13, no.3, pp.501-510, May 1998.

[7] Qian Jinrong, Zhao Qun, F.C. Lee, "Single-stage single-switch powerfactor-correction $\mathrm{AC} / \mathrm{DC}$ converters with DC-bus voltage feedback for universal line applications," IEEE Transactions on Power Electronics, vol.13, no.6, pp.1079-1088, Nov 1998.
[8] Ivakhno, V., Zamaruiev, V. \& Ilina, O. (2013). Estimation of Semiconductor Switching Losses under Hard Switching using Matlab/Simulink Subsystem. Electrical, Control and Communication Engineering, 2(1), pp. 20-26. Retrieved 6 Aug. 2013, from doi:10.2478/ecce-2013-0003

[9] M.M. Jovanovic and Jang Yungtaek, "State-of-the-art, single-phase, active power-factor-correction techniques for high-power applications an overview," IEEE Transactions on Industrial Electronics, vol.52, no.3, pp.701-708, June 2005.

[10] Mao Hengchun, F.C.Y. Lee, D. Boroyevich and S. Hiti, "Review of high-performance three-phase power-factor correction circuits," IEEE Transactions on Industrial Electronics, vol.44, no.4, pp.437-446, Aug 1997.

[11] G. A. Belov, "Dynamic models of multiphase boost pulse converter," Electricity, vol. 6, pp. 21-28, 2010.

[12] G. A. Belov and A. V. Serebryannikov, "Calculation of power factor corrector with power transistor opening at zero current," Electricity, vol. 3, pp. 46-56, 2012.

[13] Y. A. Denisov, DC Stabilizers with Pulse-Width Modulated and Frequency Modulated Quasi-Resonant Converters. Kiev: Institute of Electrodynamics of National Academy of Sciences of Ukraine, 2001.

[14] Y. A. Denisov and A. N. Gorodny, "Steady-state characteristics of quasiresonant pulse converter with a parallel zero current switching circuit," Technical Electrodynamics, Special Issue "Power Electronics and Energy Efficiency", vol. 1, pp. 20-26, 2011.

[15] Y. Z. Tsypkin, Fundamentals of the theory of automatic systems. Moscow: Science, 1977.

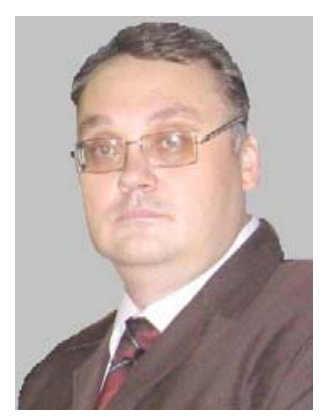

Yuriy A. Denisov received the Dipl.Eng. in industrial electronics and Ph.D. in electronics degrees from Chernihiv Technological Institute, Chernihiv, Ukraine, in 1994 and 1998 respectively, Dr.Sc. in electronics degree from Chernihiv State Technological University, Chernihiv, Ukraine, in 2006.

$\mathrm{He}$ is a Full Professor in electronics since 2008 and Head of the Department of Industrial Electronics, Chernihiv State Technological University, Chernihiv, Ukraine. He has authored over 80 published papers on power converters including several patents. His research interests are devoted to design and calculation of precision DC voltage stabilization systems.

$\mathrm{He}$ is a Vice Head of the Academic Council K 79.051.03 for $\mathrm{PhD}$ theses defenses (Chernihiv State Technological University, Chernihiv, Ukraine) and a Member of the Academic Council D 26.187.01 (Institute of Electrodynamics, Kiev, Ukraine).

Postal address: Department of Industrial Electronics, Chernihiv State Technological University, Shevchenko street, 95, Chernihiv, 14027, Ukraine. E-mail: den_1td@mail.ru

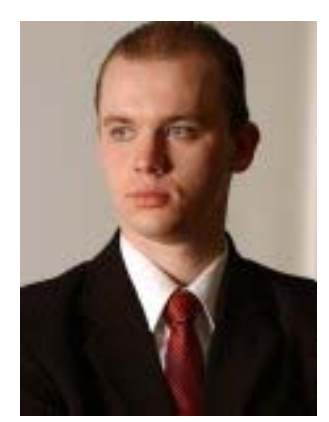

Serhii A. Stepenko (S'13) received the B.Sc. and M.Sc. degrees in industrial electronics from Chernihiv State Technological University, Chernihiv, Ukraine, in 2010 and 2011 respectively.

$\mathrm{He}$ is presently an Assistant at the Department of Industrial Electronics, Chernihiv State Technological University, Chernihiv, Ukraine, where he is currently working toward the Ph.D. degree. His Ph.D. thesis is devoted to intelligent regulation in energy efficient voltage converters. He has authored over 20 published papers including articles in the international conferences proceedings, Ukrainian and foreign scientific journals and papers indexed by IEEE Xplore, Scopus and Google Scholar. His research interests include power electronics, renewable energy, intelligent systems and FPGAs.

He has an IEEE Student Membership since 2013. He is a Member of Scientific Council of Chernihiv State Technological University, representing the rights of postgraduates.

Postal address: Department of Industrial Electronics, Chernihiv State Technological University, Shevchenko street, 95, Chernihiv, 14027, Ukraine. E-mail: stepenko.sergey@gmail.com 